Article

\title{
$N$-Iodosuccinimide as a Precatalyst for Direct Cross-Coupling of Alcohols with $C$-Nucleophiles under Solvent-Free Reaction Conditions
}

\author{
Njomza Ajvazi 1,*(D) and Stojan Stavber 1,2 \\ Jožef Stefan International Postgraduate School, Jamova 39, 1000 Ljubljana, Slovenia; stojan.stavber@ijs.si \\ 2 Jožef Stefan Institute, Jamova 39, 1000 Ljubljana, Slovenia \\ * Correspondence: njomza.ajvazi@rezonanca-rks.com; Tel.: +383-44-258-553
}

Received: 19 June 2020; Accepted: 30 July 2020; Published: 1 August 2020

\begin{abstract}
C}-\mathrm{C}$ bond formation is one of the most important implements in synthetic organic chemistry. In pursuit of effective synthetic routes functioning under greener pathways to achieve direct $\mathrm{C}-\mathrm{C}$ bond formation, we report $\mathrm{N}$-iodosuccinimide (NIS) as the most effective precatalyst among the $\mathrm{N}$-halosuccinimides (NXSs) for the direct cross-coupling of benzyl alcohols with C-nucleophiles under solvent-free reaction conditions (SFRC). The protocol is metal-free, and air- and water-tolerant, providing a large-scale synthesis with almost quantitative yields.
\end{abstract}

Keywords: alcohols; $N$-iodosuccinimide; $\mathrm{C}-\mathrm{C}$ coupling; solvent-free reactions; green chemistry

\section{Introduction}

$\mathrm{C}-\mathrm{C}$ bond formation is one of the most significant approaches for the synthesis of complex organic compounds such as pharmaceuticals and agrochemicals [1]. Alcohols are readily available and one of the most abundant organic compounds. Their employment for direct cross-coupling reactions with other partners is highly desirable, producing water as the only by-product, making the protocol environmentally benign. Since hydroxyl moieties are a weak leaving group, often an additional activation is unavoidable [2].

The hydroxyl group's activation employing a substoichiometric amount of Brønsted acids, metal ions, Lewis/Brønsted acid combinations, or other promoters has been shown by several excellent reviews [1,3-9] and recent advanced related reports [10-14]. Nevertheless, the necessity of hazardous or costly reagents, environmentally unfriendly solvents, multiple-step synthesis, a high amount of the catalyst, or high temperatures make such a synthetic procedure less preferable than the green chemical standpoint. Therefore, planning organic reactions and processes following the principles of green chemistry [15] is one of the main challenges in organic synthesis. As a result, solvent-free synthetic methods have attracted significant interest not only in laboratory synthesis but also in the chemical industry due to their simplicity and cost-efficiency. With this in mind, it is becoming essential to develop efficient, selective, and environmentally benign catalytic systems for direct dehydrative C-C coupling.

Recently, we presented the introduction of $\mathrm{N}$-halosuccinimides (NXSs) (chloro, bromo, and iodo), as the catalyst for the transformation of organic compounds bearing the hydroxyl functional group in one pot, constructing new carbon-carbon or carbon-heteroatom bonds [16]. $N$-iodosuccinimide (NIS) in substoichiometric amounts was favored as the most effective and selective catalyst among the NXSs. In continuation of our research on developing greener synthetic transformation [17-19] herein, we reported the expanding role of NIS as a non-metal, commercially available, and eco-friendly precatalyst for direct dehydrative $\mathrm{C}-\mathrm{C}$ coupling between various benzyl alcohols and different type of 
electron-rich compounds including: $\beta$-diketones, phenyl substituted alkene, tertiary benzyl alcohols bearing a vicinal hydrogen atom as alkene precursors, and heteroatom benzocyclenes selectively and efficiently into the corresponding products, under solvent-free reaction conditions.

\section{Results and Discussion}

The coupling of diphenylmethanol 1 and 1,3-diphenylpropane-1,3-dione $\mathbf{2}$ was chosen as a model reaction to employ NIS as the catalyst in direct cross-coupling of $\beta$-diketones and alcohols and to study the effects of different reaction conditions. Initially, the impact of different solvents on the conversion of $\mathbf{1}$ with $\mathbf{2}$ was studied, where it seemed to be inconvenient for the conversion of $\mathbf{1}$ with $\mathbf{2}$, while solvent-free reaction conditions were critical for quantitative transformation, and the results are shown in the Supplementary Materials (Table S1).

In search of optimal reaction conditions, different parameters, including the loading of the NIS as the catalyst, reaction time, reaction temperature for the reaction of diphenylmethanol 1 with 2 under solvent-free conditions, and the reactivity of alcohols, were first studied, and the results are given in the Supplementary Materials (Tables S2-S5). It could be seen that under the mentioned optimal reaction conditions, the reaction successfully proceeded only in the case of secondary benzyl alcohol 1 (entry 1, Table 1). In contrast, no reaction occurred with unsubstituted benzyl alcohol 5 as the type of primary alcohol, due to lower reactivity, and trityl alcohol $\mathbf{6}$ as the type of tertiary alcohol, which could be explained by the steric effect [16].

By further investigation of the crucial reaction parameters, such as the structure of the $ß$-dicarbonyl compound, the structure of alcohol, and loading of the catalyst, showed us the real value of this methodology. Thus, the effect of the structure of $ß$-diketone on the course of the reaction with $\mathbf{1}$ as the most reactive benzyl alcohol derivative was studied. We selected benzoylacetone as a further candidate where the addition of NIS as the catalyst was observed to promote effectively and selectively the conversion of 1 with benzoylacetone $\mathbf{7}$ under solvent-free reaction conditions (SFRC) into the corresponding product 8 (entry 5 , Table 1 ).

To increase the yield of the corresponding product 8 , different concentrations of the catalyst and variations of reaction temperatures were applied, and the results are shown in the Supplementary Materials (Tables S6 and S7). In the absence of a precatalyst, no reaction occurred.

Given the mentioned experience, it seemed that acetylacetone could be the next instructive substrate for realizing the effect of the structure of a B-dicarbonyl compound to the course of the reaction, where the addition of NIS as the catalyst was found to provide efficient and selective transformation of $\mathbf{1}$ with acetylacetone $\mathbf{9}$ under SFRC into the corresponding product $\mathbf{1 0}$ (entry 7, Table 1). To increase the yield of the corresponding product 10, different concentrations of the catalyst were applied, and the results are presented in the Supplementary Materials (Table S8). In the absence of a catalyst, no reaction took place.

According to these results, the most reactive of $\beta$-dicarbonyl compounds was 1,3-diphenylpropane1,3-dione 2, optimal loading of NIS as the catalyst was $1 \mathrm{~mol} \%$, reaction temperature was $70-75{ }^{\circ} \mathrm{C}$, and reaction time was $24 \mathrm{~h}$. Moreover, during the optimization of reaction conditions, we found that varying the temperature from $70-75^{\circ} \mathrm{C}$ to $100-105^{\circ} \mathrm{C}$ for the same reaction shortened the reaction time $(6 \mathrm{~h})$ and provided excellent yield.

We believed that the reaction's key point was the degree of enolization of $\beta$-diketone on one side, and stability of the carbocationic intermediate formed from the alcohol on another side. A higher degree of enolization $\left(\mathrm{pK}_{\mathrm{E}}\right.$ ) caused more potent and particular transformation into the desired coupling between B-dicarbonyl and alcohol, and a more stabilized carbocationic intermediate caused more efficient and selective product formation.

Through solvation, stability and reactivity of enolates can be controlled. Enolates are more stable in polar protic solvents, such as water, than in polar aprotic solvents, such as dimethyl sulfoxide [20].

Encouraged by these promising results, we checked the scope of the reaction system by applying the obtained optimal reaction conditions on cross-coupling reactions of $ß$-dicarbonyl compounds with 
different alcohols catalyzed by NIS under SFRC. The results of reactions carried out under SFRC are collected in Table 1.

Table 1. Reactions of $\mathrm{B}$-dicarbonyl compounds with different alcohols catalyzed by $\mathrm{N}$-iodosuccinimide (NIS) under solvent-free reaction conditions (SFRC) ${ }^{\mathrm{a}}$.<smiles>[R]c1ccc(C([R])(O)c2ccc(C)cc2)cc1</smiles><smiles>[R]c1ccc(C([R])([R])C(C(C)=O)C(C)=O)cc1</smiles>

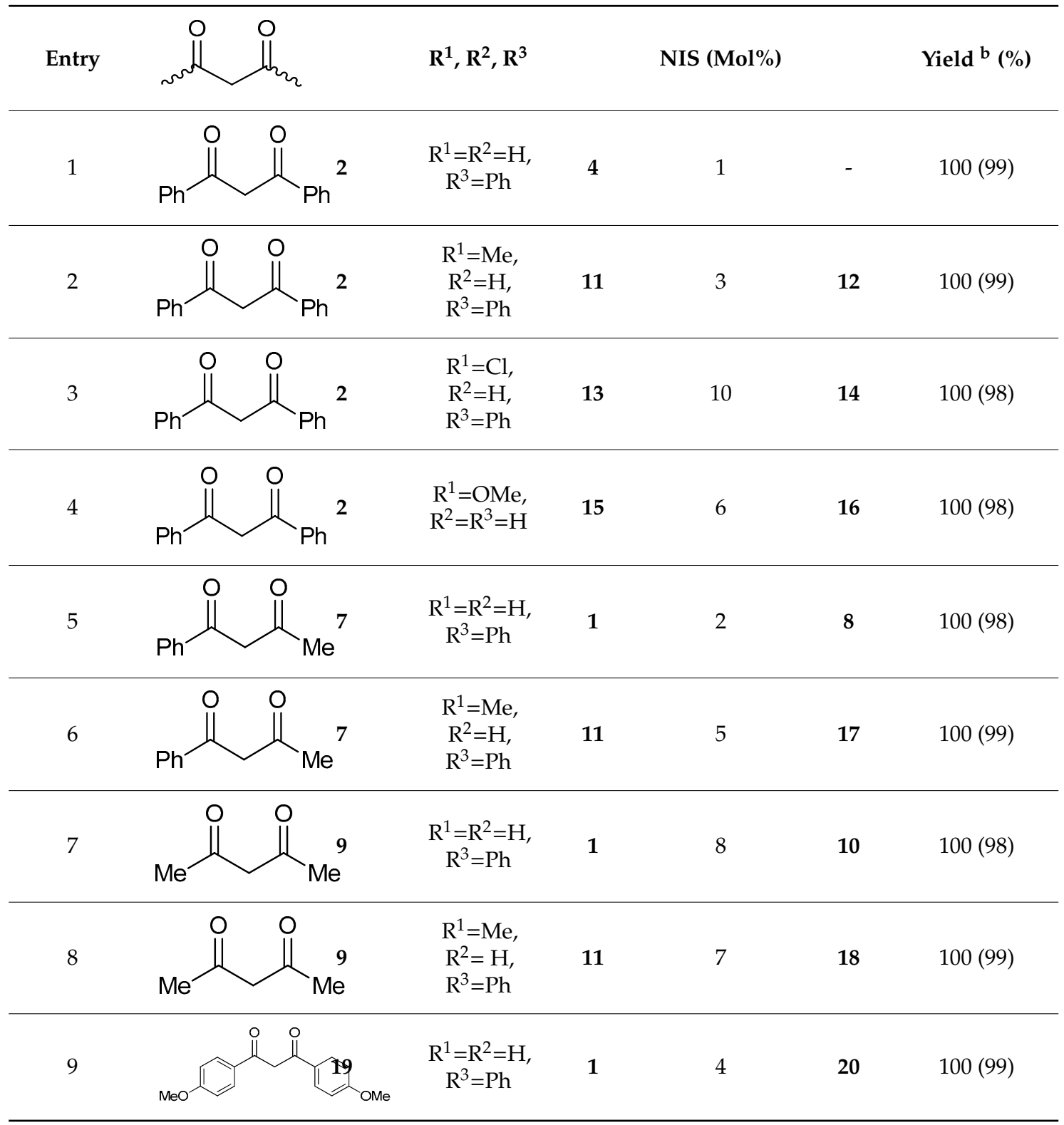

a Reaction conditions: alcohols ( $0.5 \mathrm{mmol})$, diketones ( $0.5 \mathrm{mmol})$, NIS (1-10 mol\%), 70-75 ${ }^{\circ} \mathrm{C}, 23-24 \mathrm{~h} .{ }^{\mathrm{b}}$ Determined from ${ }^{1} \mathrm{H}$ NMR spectra of isolated crude reaction mixtures; yield calculated relative to alcohol; values in parentheses are isolated yields.

Effective transformation with $\beta$-diketone 2 was observed in the reaction with additional classes of secondary benzyl alcohols, including derivatives of diphenylmethanol bearing electron-donating or 
electron-withdrawing substituents on the aromatic rings $\mathbf{1 1}$ and 13, and established the quantitative formation of the corresponding products 12 and $\mathbf{1 4}$ (entries 2 and 3, Table 1).

Furthermore, we checked the reaction of the primary benzyl alcohol bearing electron-donating group with 2. 4-methoxybenzyl alcohol 15 was efficiently and selectively converted into the 2-(4-methoxybenzyl)1,3-diphenylpropane-1,3-dione $\mathbf{1 6}$ (entry 4, Table 1). We further investigated the corresponding reactions of alcohols with benzoylacetone 7 . In the case of the reaction of primary benzyl alcohol $\mathbf{5}$ with $\mathbf{7}$ in the presence of NIS as the catalyst under solvent-free conditions, no transformation took place. Effective and selective transformation with 7 was observed in the reaction with phenyl( $p$-tolyl)methanol 11, providing the quantitative formation of the corresponding product $\mathbf{1 7}$ (entry 6, Table 1). No transformation was observed with sterically bulkier triphenylmethanol 6 with 7 catalyzed by NIS under SFRC.

Moreover, we investigated the corresponding reactions of alcohols with acetylacetone 9 . In the case of the reaction of unsubstituted benzyl alcohol 5 with 9 catalyzed by NIS under SFRC, no transformation took place. Effective transformation of phenyl( $p$-tolyl)methanol 11 with $\mathbf{9}$ was observed and established the quantitative formation of the corresponding product 18 (entry 8, Table 1). No transformation was observed in the case of triphenylmethanol 6 with 9 catalyzed by NIS under SFRC. Additionally, the effective transformation was obtained between 1,3-bis(4-methoxyphenyl)propane-1,3-dione 19 and $\mathbf{1}$, furnishing the respective product 20 in excellent yield (entry 9, Table 1).

To establish the synthetic value of the reported methodology, the following reaction was performed on the $10 \mathrm{mmol}$ scales. Coupling of $\mathbf{1}$ with $\mathbf{2}$ under the above-mentioned optimal reaction conditions afforded the corresponding product 4 in quantitative yield (99\%).

Inspired by these results, we investigated NIS's efficiency as the catalyst for the direct coupling of phenyl substituted alkene with secondary benzyl alcohol under solvent-free conditions. NIS, as an effective catalyst, was observed to promote the direct coupling of 1,1 diphenylethene 21 with phenyl ( $p$-tolyl) methanol $\mathbf{1 1}$ under SFRC, producing the corresponding substituted alkene $\mathbf{2 3}$ in nearly quantitative yield (Scheme 1).

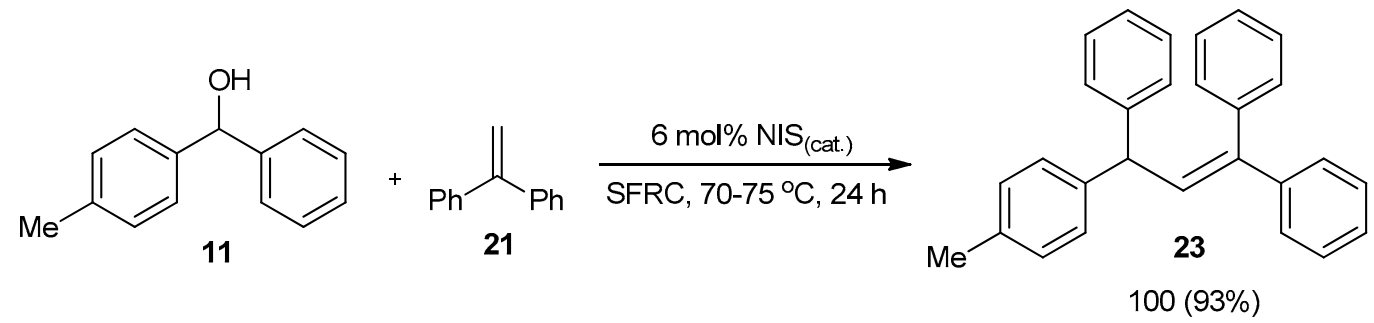

Scheme 1. Reaction of phenyl( $p$-tolyl)methanol 11 with 1,1 diphenylethene 21 catalyzed by NIS under SFRC.

It is well-known that alkenes can be readily achieved by dehydration of tertiary alcohols bearing a vicinal hydrogen atom. Inspired by this finding, we performed the reaction between 1,1-diphenylethanol 24 and 1 catalyzed by NIS under SFRC. We observed that the direct coupling of two alcohols, 1 and 24, forming the corresponding product 22 as the major product took place, accompanied by a trace amount of side products identified as benzophenone and dimeric ether 3, (Scheme 2).

Electron-rich benzocyclenes with a general structure $(X=N A c, O)$ were chosen as potential partners for the NIS-promoted reactions with benzyl alcohols under SFRC. The reaction of diphenylmethanol 1 with 1,2-dihydronaphthalene 25 catalyzed by NIS under SFRC performed the corresponding substituted alkene 26, accompanied by a small amount of dimeric ether $\mathbf{3}$, was observed. Different parameters such as the loading of the NIS as the catalyst and the reaction temperature for the reaction of 1 with 1,2-dihydronaphthalene 25 under SFRC were first studied, and the results are shown in the Supplementary Materials (Tables S8 and S9). Moreover, during the optimization of reaction conditions, we found that equimolar of $\mathbf{1}$ and $\mathbf{2 5}$ provided the best result in terms of efficiency and selectivity. 


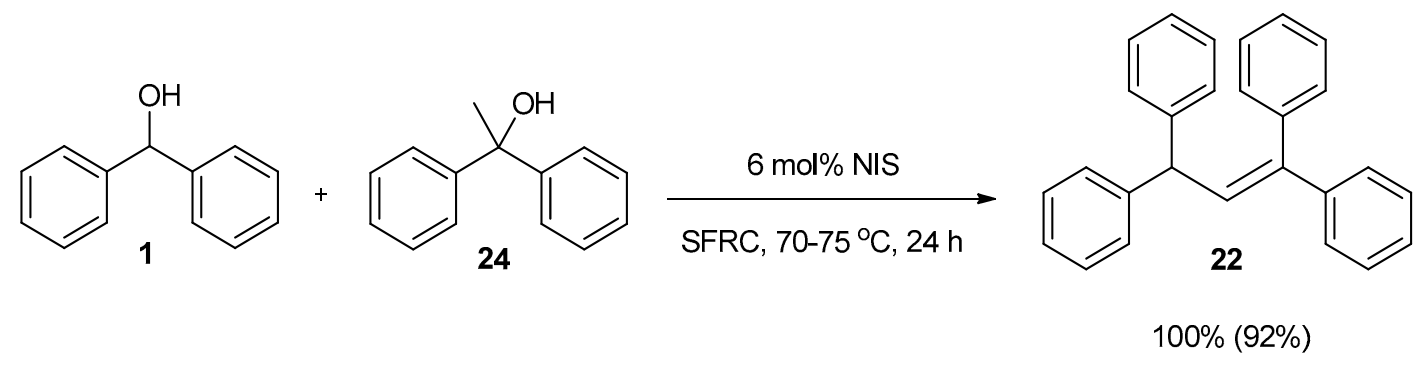

Scheme 2. Direct cross-coupling of diphenylmethanol 1 and 1,1-diphenylethanol 24 catalyzed by NIS under SFRC.

To verify the presumption that the symmetric ether 3 could be the intermediate of the crosscoupling [21], we independently performed the reaction between symmetric ether 3 as a source of benzylic cation, which was synthesized from 1 catalyzed by NIS $(2 \mathrm{~mol} \%)$ at $70-75^{\circ} \mathrm{C}$, for $3.5 \mathrm{~h}$ under SFRC. Then, alkylation of the dimeric ether 3 by dihydronaphthalene 25 took place, providing the corresponding product $\mathbf{2 6}$ in quantitative yield. Another possible pathway might be a direct reaction of carbocation derived from alcohol $\mathbf{1}$ and followed by nucleophile displacement (Scheme 3).
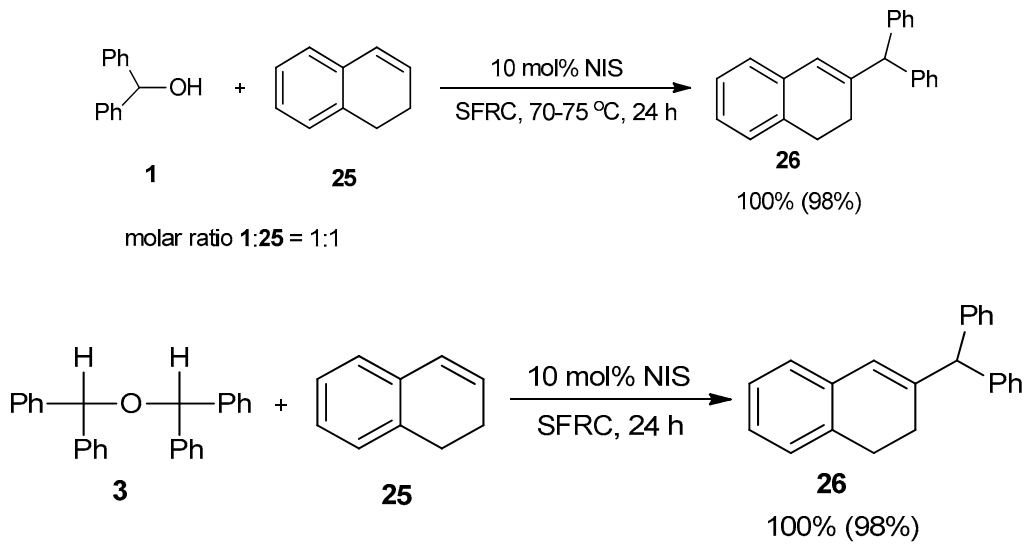

Scheme 3. Control reactions.

According to the results of the control reaction shown in Scheme 3, a potential explanation suggests a decomposition of the precatalyst NIS to form HOI in alcoholic reaction media, which could be responsible for NIS's mediation activity [22-25]. The $\mathrm{X}_{2}$ forms HOI and $\mathrm{HI}$ in aqueous media, providing the regeneration of HOI for the following catalysis. Therefore, it was reasonable to assume that the water produced as the only by-product of the process could act as a supporting factor in the acceleration of the reaction, since no conversion was observed in the reaction between diphenylmethanol 1 and 1,3-diphenylpropane-1,3-dione $\mathbf{2}$ catalyzed by NIS in aqueous media. The presumption that NIS was actually a precatalyst forming HOI, $\mathrm{I}_{2}$, and protons during the process, which might catalyze nucleophilic substitutions, showed to be reasonable $[16,26]$.

The generality of this methodology for $\mathrm{C}-\mathrm{C}$ hetero coupling approaches with heteroatom benzocyclenes 27 and 29 with $\mathbf{1}$, catalyzed by NIS under SFRC, was further examined. The results of the reactions carried out under SFRC are collected in Table 2. NIS catalyzed the direct coupling of 1 with an $\mathrm{N}$-based heteroarene such as 1-acetylindole $\mathbf{2 3}$ under SFRC, affording C-3 alkylated product in high yield (28, entry 1 , Table 2). The direct coupling of $\mathbf{1}$ with an $O$-containing heteroarene such as 2,3-benzofuran 29 underwent smooth coupling to provide the C-2 alkylated product in good yield (30, entry 2, Table 2). It could be seen that the electrophilic substitution in 1-acetylindole 27 took place at $\mathrm{C}-3$ and not at C-2. This orientation could be explained by comparing the stability of the carbocation resulting from the electrophilic attack at C-2, which was less favorable than the carbocation from 
the electrophilic attack at C-3. The electrophilic substitution at C-2 in benzofuran 29 could also be explained in the same way.

Table 2. Reactions of diphenylmethanol 1 with heteroarenes $\mathbf{2 7 - 2 9}$ catalyzed by NIS under SFRC ${ }^{\text {a }}$

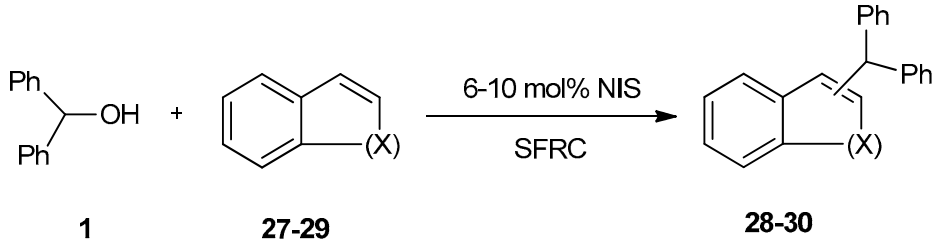

\begin{tabular}{llllll}
\hline Entry & $\mathbf{X}$ & Yield (\%) & \\
\hline
\end{tabular}

a Reaction conditions: diphenylmethanol 1 ( $0.5 \mathrm{mmol})$, heteroarenes $27-29(0.4 \mathrm{mmol}), 70-75{ }^{\circ} \mathrm{C}, 24 \mathrm{~h} .{ }^{\mathrm{b}}$ Isolated products; yields calculated relative to heteroatom benzocyclenes.

To gain clearer insight into NIS's thermal stability, thermal gravimetric analysis (TGA) on the precatalyst was carried out. It was observed that the degradation did not happen to the precatalyst at temperatures $25-200{ }^{\circ} \mathrm{C}[16]$.

\section{Materials and Methods}

All starting materials and NIS were commercially available and used without further purification (Merck, Darmstadt, Germany; Sigma Aldrich, St. Louis, MO, USA). Reactions were performed in $4 \mathrm{~mL}$ screw-capped vials. All reactions were observed by thin-layer chromatography (TLC, mobile phase: dichloromethane/hexane 9:1) (with silica gel/TLC cards; DC-Alufolien-Kieselgel, Sigma-Aldrich, St. Louis, MO, USA) and visualized by UV lamp (254 nm, Camag, Muttenz, Switzerland). ${ }^{1} \mathrm{H}$ and ${ }^{13} \mathrm{C}$ NMR spectra were measured on Varian INOVA 300 NMR instrument, using a solution of $\mathrm{CDCl}_{3}$ as the solvent with $\mathrm{SiMe}_{4}$ as an internal reference standard. Melting points were measured by Buchi 535 equipment. Purification of a few products was performed by column chromatography (CC) using silica gel 60 (particle size: $0.063-0.200 \mathrm{~mm}$ ).

General procedure for new $\mathrm{C}-\mathrm{C}$ bond construction through $\beta$-diketone or electron-rich $\mathrm{C}=\mathrm{C}$ bonds in organic molecule catalyzed by NIS on half mmol scale:

The mixture of benzyl alcohol $(0.5 \mathrm{mmol}), \beta$-diketone, or alkene $(0.5 \mathrm{mmol})$ and NIS $(1-10 \mathrm{~mol} \%)$, after being powdered in a mortar in the case of solid-state reactants, was transferred to a $4 \mathrm{~mL}$ screw-capped vial and heated at $70-75{ }^{\circ} \mathrm{C}$ for $24 \mathrm{~h}$. TLC monitored the progress of the reaction mixture. The crude reaction mixture was cooled down to room temperature and diluted with a mixture of $(3 \times$ $5 \mathrm{~mL}$ EtOAc), $\left(2 \times 3 \mathrm{~mL}\right.$ saturated $\left.\mathrm{Na}_{2} \mathrm{~S}_{2} \mathrm{O}_{3}\right),\left(2 \times 3 \mathrm{~mL}\right.$ saturated $\left.\mathrm{NaHCO}_{3}\right)$, and $(2 \times 5 \mathrm{~mL}$ distilled water). The organic phase was dried $\mathrm{Na}_{2} \mathrm{SO}_{4}$, and the organic solvent was removed under reduced pressure, leaving the resultant residue.

The scaled-up procedure for the synthesis of 2-benzhydryl-1,3-diphenylpropane-1,3-dione 4 catalyzed by NIS:

The mixture of $\beta$-diketone 2 (10 mmol, $1.8423 \mathrm{~g})$, diphenylmethanol $1(10 \mathrm{mmol}, 2.2425 \mathrm{~g})$, and NIS ( $1 \mathrm{~mol} \%, 0.1 \mathrm{~mol}, 22.5 \mathrm{mg}$ ), which was powdered in a mortar, was transferred to a $20 \mathrm{~mL}$ screw-capped glass scintillation vial and heated at $70-75{ }^{\circ} \mathrm{C}$ for $24 \mathrm{~h}$. TLC followed the progress of the reaction 
mixture. Upon completion of the reaction, the mixture was cooled to room temperature. Finally, the crude reaction mixture was washed with hot water and filtered by vacuum filtration to obtain a pure product in almost quantitative yield (white solid, m.p. $220-223^{\circ} \mathrm{C}, 3.9 \mathrm{~g}, 99 \%$ ).

\section{Conclusions}

In summary, simple, efficient, selective, and easily scalable methodology for $\mathrm{C}-\mathrm{C}$ bond formation through the direct cross-coupling of various benzyl alcohols with $\beta$-diketones, heteroatom benzocyclenes, phenyl substituted alkenes, or tertiary benzyl alcohols bearing a vicinal hydrogen atom as alkene precursors employing NIS, an environmentally friendly and metal-free precatalyst, under SFRC was developed. The reactivity of B-dicarbonyl compounds was found to be the function of their enolisability, whereas primary benzyl alcohol targets needed strong activation of the ring; a comprehensive range of secondary benzyl alcohols gave efficient coupling, whereas tertiary benzyl alcohols without vicinal hydrogen atoms were found as inconvenient targets. Benzylation of the position C-2 was observed in the case of 1,2-dihydronaphthalene 25 and 2,3-benzofuran 29, whereas C-3 was benzylated in 1-acetylindole 27.

Supplementary Materials: The following are available online at http://www.mdpi.com/2073-4344/10/8/850/s1, detailed experimental data, ${ }^{1} \mathrm{H}-\mathrm{NMR}$ and ${ }^{13} \mathrm{C}-\mathrm{NMR}$ spectra of isolated final products.

Author Contributions: Conceptualization, S.S.; formal analysis, N.A. and S.S.; Investigation, N.A. and S.S.; methodology, N.A. and S.S.; writing — original draft, N.A. and S.S.; writing-review and editing, N.A. and S.S. All authors have read and agreed to the published version of the manuscript.

Funding: This research was funded by Slovene Human Resources Development and Scholarship Fund (contract: 11011-9/2011), Slovenian Research Agency (contract: Programme P1-0134).

Acknowledgments: The authors are grateful to the Slovenian NMR Centre at the National Institute of Chemistry, Ljubljana, Slovenia.

Conflicts of Interest: The authors declare no conflict of interest. The funders had no role in the design of the study; in the collection, analyses, or interpretation of data; in the writing of the manuscript; or in the decision to publish the results.

\section{References}

1. Kumar, R.; Van Der Eycken, E.V. Recent approaches for $\mathrm{C}-\mathrm{C}$ bond formation via direct dehydrative coupling strategies. Chem. Soc. Rev. 2013, 42, 1121-1146. [CrossRef] [PubMed]

2. Yasuda, M.; Saito, T.; Ueba, M.; Baba, A. Direct substitution of the hydroxy group in alcohols with silyl nucleophiles catalyzed by indium trichloride. Angew. Chem. Int. Ed. 2004, 43, 1414-1416. [CrossRef] [PubMed]

3. Emer, E.; Sinisi, R.; Capdevila, M.G.; Petruzziello, D.; De Vincentiis, F.; Cozzi, P.G. ChemInform Abstract: Direct nucleophilic SN1-type reactions of alcohols. Eur. J. Org. Chem. 2011, 2011, 647-666. [CrossRef]

4. Chen, L.; Yin, X.-P.; Wang, C.-H.; Zhou, J. Catalytic functionalization of tertiary alcohols to fully substituted carbon centres. Org. Biomol. Chem. 2014, 12, 6033-6048. [CrossRef] [PubMed]

5. Uchuskin, M.G.; Makarov, A.S.; Butin, A.V. Catalytic Alkylation of furans by $\pi$-activated alcohols (Review). Chem. Heterocycl. Compd. 2014, 50, 791-806. [CrossRef]

6. Chaskar, A.; Murugan, K. Direct allylation of alcohols using allyltrimethylsilane: A move towards an economical and ecological protocol for C-C bond formation. Catal. Sci. Tech. 2014, 4, 1852-1868. [CrossRef]

7. Shang, X.-J.; Liu, Z.-Q. Iron-catalyzed alkylation of alkenes and alkynes using alcohols as the alkylating reagent. Synthesis 2015, 47, 1706-1708. [CrossRef]

8. Moran, J.; Dryzhakov, M.; Richmond, E. Recent advances in direct catalytic dehydrative substitution of alcohols. Synthesis 2016, 48, 935-959. [CrossRef]

9. Ajvazi, N.; Stavber, S. Alcohols in direct carbon-carbon and carbon-heteroatom bond-forming reactions: Recent advances. Arkivoc 2018, 2018, 288-329. [CrossRef]

10. Yang, G.-P.; Jiang, N.; Huang, X.-Q.; Yu, B.; Hu, C.-W. Non-corrosive heteropolyacid-based recyclable ionic liquid catalyzed direct dehydrative coupling of alcohols with alcohols or alkenes. Mol. Catal. 2019, 468, 80-85. [CrossRef] 
11. Chevella, D.; Macharla, A.K.; Kodumuri, S.; Banothu, R.; Gajula, K.S.; Amrutham, V.; Gennadievna, G.N.; Nama, N.; Durgaiah, C.; Kumar, M.A.; et al. Synthesis of internal olefins by direct coupling of alcohols and olefins over Mo $\beta$ zeolite. Catal. Commun. 2019, 123, 114-118. [CrossRef]

12. Bhattacharjee, P.; Bora, U. Molecular iodine-catalyzed selective C-3 benzylation of indoles with benzylic alcohols: A greener approach toward benzylated indoles. ACS Omega 2019, 4, 11770-11776. [CrossRef] [PubMed]

13. Böldl, M.; Fleischer, I. Dehydrative coupling of benzylic alcohols catalyzed by Brønsted Acid/Lewis Base. Eur. J. Org. Chem. 2019, 2019, 5856-5861. [CrossRef]

14. Čebular, K.; Stavber, S. Molecular iodine as a mild catalyst for cross-coupling of alkenes and alcohols. Pure Appl. Chem. 2018, 90, 377-386. [CrossRef]

15. Anastas, P.T.; Warner, J.C. Green Chemistry: Theory and Practice; Oxford University Press: New York, NY, USA, 1998.

16. Ajvazi, N.; Stavber, S. N-halosuccinimides as precatalysts for C-, N-, O-, and X-nucleophilic substitution reactions of alcohols under mild reaction conditions. Catalysts 2020, 10, 460. [CrossRef]

17. Stavber, G.; Iskra, J.; Zupan, M.; Stavber, S. aerobic oxidative iodination of organic compounds with iodide catalyzed by sodium nitrite. Adv. Synth. Catal. 2008, 350, 2921-2929. [CrossRef]

18. Stavber, G.; Iskra, J.; Zupan, M.; Stavber, S. Aerobic oxidative iodination of ketones catalysed by sodium nitrite "on water" or in a micelle-based aqueous system. Green Chem. 2009, 11, 1262. [CrossRef]

19. Stavber, G.; Stavber, S. Towards greener fluorine organic chemistry: Direct electrophilic fluorination of carbonyl compounds in water and under solvent-free reaction conditions. Adv. Synth. Catal. 2010, 352, 2838-2846. [CrossRef]

20. Eames, J. Acid-Base Properties of Enols and Enolates. In PATAI'S Chemistry of Functional Groups; John Wiley \& Sons, Ltd.: Hoboken, NJ, USA, 2009.

21. Chun, S.; Chung, Y.K. Silver/NBS-catalyzed synthesis of $\alpha$-alkylated aryl ketones from internal alkynes and benzyl alcohols via ether intermediates. Org. Lett. 2018, 20, 5583-5586. [CrossRef]

22. Bulfield, D.; Huber, S.M. Halogen Bonding in Organic Synthesis and Organocatalysis. Chem. A Eur. J. 2016, 22, 14434-14450. [CrossRef]

23. Breugst, M.; Von Der Heiden, D. Mechanisms in Iodine Catalysis. Chem. A Eur. J. 2018, 24, 9187-9199. [CrossRef] [PubMed]

24. Clark, T.; Hennemann, M.; Murray, J.S.; Politzer, P. Halogen bonding: The $\sigma$-hole. J. Mol. Model. 2006, 13, 291-296. [CrossRef] [PubMed]

25. Politzer, P.; Murray, J.S.; Clark, T. Halogen bonding: An electrostatically-driven highly directional noncovalent interaction. Phys. Chem. Chem. Phys. 2010, 12, 7748-7757. [CrossRef] [PubMed]

26. Čebular, K.; Božić, B.; Stavber, S. 1,3-dibromo-5,5-dimethylhydantoin as a precatalyst for activation of carbonyl functionality. Molecules 2019, 24, 2608. [CrossRef] [PubMed] 